\title{
Photon's Structure of Motion
}

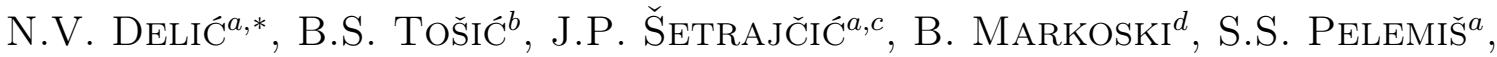 \\ V.D. SAJFERT ${ }^{d}$ AND V.M. ZORIĆ ${ }^{a}$ \\ ${ }^{a}$ Department of Physics, Faculty of Sciences, University of Novi Sad, Vojvodina, Serbia \\ ${ }^{b}$ Vojvodina Academy of Sciences and Arts, Novi Sad, Vojvodina, Serbia \\ ${ }^{c}$ Academy of Sciences and Arts in Banja Luka, Republic of Srpska, BiH \\ ${ }^{d}$ Technical Faculty in Zrenjanin, University of Novi Sad, Vojvodina, Serbia \\ ${ }^{e}$ Faculty of Tecnology in Zvornik, University of East Sarajevo, Republic of Srpska, BiH
}

\begin{abstract}
A free photon Hamiltonian is linearized using Pauli's matrices. Based on the correspondence of Pauli's matrices kinematics and the kinematics of spin operators, it has been proved that a free photon integral of motion is a sum of orbital momentum and spin momentum for a half-one spin. Linearized Hamiltonian represents a bilinear form of products of spin and momentum operators. Unitary transformation of this form results in an equivalent Hamiltonian, which has been analyzed by the method of Green's functions. The evaluated Green function has given possibility for interpretation of photon reflection as a transformation of photon to antiphoton with energy change equal to double energy of photon and for spin change equal to Dirac's constant. Since photon is relativistic quantum object the exact determining of its characteristics is impossible. It is the reason for series of experimental works in which photon orbital momentum, which is not integral of motion, was investigated. The exposed theory was compared to the mentioned experiments and in some elements the satisfactory agreement was found.
\end{abstract}

PACS numbers: 14.70.Bh, 33.60.+q, 42.50.Ar, 42.50.Dr

\section{Introduction}

The fact that photon Hamiltonian is not a linear operator has a set of consequences that have not been studied sufficiently so far. The main reason is that photon characteristics have been mainly examined by means of Klein-Gordon's equation [1], which represents eigenproblem of photon Hamiltonian square.

In this paper we shall linearize photon Hamiltonian and examine some of photon characteristics which follow from linearized Hamiltonian. The analogy with Dirac's approach to the problem of electrons will be used [2]. In the first part of the paper integrals of motion of free photon will be examined and it will be shown that the orbital angular momentum of photon is not the integral of motion. It will be shown that the integral of motion is total angular momentum being the sum of orbital one and spin momentum. The unitary transformation of linearized photon Hamiltonian, which leads to equivalent Hamiltonian suitable for application the Green functions method, will be made in the second part of the paper.

The discussion of obtained results and their comparison to the experimental data will be done at concluding part.

\footnotetext{
* corresponding author; e-mail:

alannica@gmail.com (bora@im.ns.ac.yu)
}

\section{Linearized photon Hamiltonian}

Free photon energy is proportional to square root of the sum of the momentum components squares. If we use quantum expression approach instead of classic one, photon Hamiltonian takes the following form:

$$
\hat{H}= \pm c \sqrt{\hat{p}_{x}^{2}+\hat{p}_{y}^{2}+\hat{p}_{z}^{2}},
$$

where $\hat{p}_{j}=-\mathrm{i} \hbar \frac{\partial}{\partial x_{j}} ; j=(x, y, z)$, are the operators of photon momentum components and $c$ is velocity of light. This Hamiltonian resembles free electron Hamiltonian. The difference is that photon rest mass is equal to zero, so there is no fourth component of momentum as in the case of an electron. The main problem of quantum photon theory is the fact that Hamiltonian (2.1) is not a linear operator and this is in contradiction with the superposition principle.

This problem was first solved by Klein and Gordon, who considered the eigenproblem of its square, instead of eigenproblem of Hamiltonian (2.1). The Klein-Gordon eigenproblem was

$$
\hat{H}^{2} \Psi=E^{2} \Psi \text {. }
$$

It is obvious from (2.1) that $\hat{H}^{2}$ is a linear operator, so the main problem has been overcome in this way. Equation (2.2) is called Klein-Gordon's equation and it is still used for photons. The only problem related to KleinGordon's equation is the appearance of negative state probabilities. It has been shown [3], however, that nega- 
tive probabilities do not appear for particles and quasi-particles with zero spin and with integer spin, so this is why Klein-Gordon's approach has been maintained in free photon theory.

The second possible approach is analogous to Dirac's approach to a free electron problem [2]. Hamiltonian is linearized by converting a sum of squares into a square of a sum. In case of Hamiltonian (2.1) this procedure is formulated by the following equation:

$$
\hat{p}_{x}^{2}+\hat{p}_{y}^{2}+\hat{p}_{z}^{2}=\left(\hat{\alpha} \hat{p}_{x}+\hat{\beta} \hat{p}_{y}+\hat{\chi} \hat{p}_{z}\right)^{2},
$$

where $\hat{\alpha}, \hat{\beta}$ and $\hat{\chi}$ cannot be ordinary numbers or functions, since (2.3) cannot be fulfilled then. The conditions under which (2.3) would be fulfilled, are as follows: $\hat{\alpha}^{2}=\hat{\beta}^{2}=\hat{\chi}^{2}=1 ; \hat{\alpha} \hat{\beta}+\hat{\beta} \hat{\alpha}=0 ; \hat{\alpha} \hat{\chi}+\hat{\chi} \hat{\alpha}=0$; $\hat{\beta} \hat{\chi}+\hat{\chi} \hat{\beta}=0$. It can be easily concluded that $2 \times 2$ matrices

$$
\begin{array}{ll}
\hat{\alpha}=\left(\begin{array}{ll}
0 & 1 \\
1 & 0
\end{array}\right), \quad \hat{\beta}=\left[\begin{array}{cc}
0 & -\mathrm{i} \\
\mathrm{i} & 0
\end{array}\right], \\
\hat{\chi}=\left(\begin{array}{cc}
1 & 0 \\
0 & -1
\end{array}\right),
\end{array}
$$

fulfill above conditions. These matrices are known in literature as Pauli's matrices [1, 2, 4]. From this, photon Hamiltonian becomes a linear operator but given in the form of $2 \times 2$ matrix, i.e.

$$
\hat{H}= \pm c\left(\begin{array}{cc}
\hat{p}_{z} & \hat{p}_{x}-\mathrm{i} \hat{p}_{y} \\
\hat{p}_{x}+\mathrm{i} \hat{p}_{y} & -\hat{p}_{z}
\end{array}\right) .
$$

The fact that Hamiltonian is $2 \times 2$ square matrix requires two-component photon eigenstates in the form of rows and columns. In addition to this, every operator included in calculation with photon Hamiltonian (2.5) must be represented as $2 \times 2$ diagonal matrix. Now, the eigenproblem of Hamiltonian is written down as

$$
\begin{aligned}
& \pm c\left(\begin{array}{cc}
\hat{p}_{z} & \hat{p}_{x}-\mathrm{i} \hat{p}_{y} \\
\hat{p}_{x}+\mathrm{i} \hat{p}_{y} & -\hat{p}_{z}
\end{array}\right)\left(\begin{array}{l}
\Psi_{1} \\
\Psi_{2}
\end{array}\right) \\
& =E\left(\begin{array}{c}
\Psi_{1} \\
\Psi_{2}
\end{array}\right) .
\end{aligned}
$$

\section{Photon's integrals of motion}

We shall not deal with this eigenproblem in further. Instead of this we shall look for integrals of motion, i.e. those operators that commute with Hamiltonian (2.5). It is obvious that any function depending on momentum components represents an integral of motion, but this fact is not of physical interest.

It is of particular importance whether orbital angular momentum

$$
\hat{\boldsymbol{L}} \equiv\left(\begin{array}{cc}
\hat{\boldsymbol{L}} & 0 \\
0 & \hat{\boldsymbol{L}}
\end{array}\right), \quad \hat{\boldsymbol{L}}=\boldsymbol{r} \times \hat{\boldsymbol{p}}
$$

is photon integral of motion, since in non-relativistic quantum mechanics operator $\hat{\boldsymbol{L}}$ is integral of motion of electron [4]. If we use commutation relations for components of radius vector and the components of angular momentum: $\left[x_{i}, \hat{p}_{j}\right]=\mathrm{i} \hbar \delta_{i j} ; i, j \in(x, y, z)$ and look for commutators of orbital angular momentum components with Hamiltonian (2.5), we come to the following relations:

$$
\begin{aligned}
& {\left[\hat{L}_{x}, \hat{H}\right]= \pm \mathrm{i} \hbar c\left(\hat{p}_{z} \hat{\beta}-\hat{p}_{y} \hat{\chi}\right),} \\
& {\left[\hat{L}_{y}, \hat{H}\right]= \pm \mathrm{i} \hbar c\left(\hat{p}_{x} \hat{\chi}-\hat{p}_{z} \hat{\alpha}\right),} \\
& {\left[\hat{L}_{z}, \hat{H}\right]= \pm \mathrm{i} \hbar c\left(\hat{p}_{y} \hat{\alpha}-\hat{p}_{x} \hat{\beta}\right),}
\end{aligned}
$$

based on which it follows that orbital angular momentum is not a free photon integral of motion. It should be pointed out that signs in (3.2) are obtained on the basis of obvious symmetry properties $\hat{H}(-\boldsymbol{r})=\hat{H}(\boldsymbol{r})$ and $\boldsymbol{L}(-\boldsymbol{r})=\boldsymbol{L}(\boldsymbol{r})$, where $\boldsymbol{r}$ is radius vector. In order to find some rotation characteristics that commute with a free photon Hamiltonian, we shall first show that commutation relations for matrices $\hat{\alpha}, \hat{\beta}$ and $\hat{\chi}$ are: $[\hat{\alpha}, \hat{\beta}]=2 \mathrm{i} \hat{\chi}$; $[\hat{\chi}, \hat{\alpha}]=2 \mathrm{i} \hat{\beta} ;[\hat{\beta}, \hat{\chi}]=2 \mathrm{i} \hat{\alpha}$. Very similar to these relations are the commutation relations for spin components: $\left[\hat{S}_{x}, \hat{S}_{y}\right]=\mathrm{i} \hbar \hat{S}_{z} ;\left[\hat{S}_{z}, \hat{S}_{x}\right]=\mathrm{i} \hbar \hat{S}_{y} ;\left[\hat{S}_{y}, \hat{S}_{z}\right]=\mathrm{i} \hbar \hat{S}_{x}$. Comparing this we can establish the correspodence between spin operators and Pauli's matrices

$$
\hat{S}_{x}=\frac{\hbar}{2} \hat{\alpha}, \quad \hat{S}_{y}=\frac{\hbar}{2} \hat{\beta}, \quad \hat{S}_{z}=\frac{\hbar}{2} \hat{\chi} .
$$

We shall now look for a commutator of component $\hat{J}_{x}$ of total momentum with photon Hamiltonian, i.e. with $\hat{H}(\boldsymbol{r})$. Using upper signs in formulae (3.2) we obtain

$$
\begin{aligned}
& {\left[\hat{J}_{x}, \hat{H}(\boldsymbol{r})\right]=\left[\left(\hat{L}_{x}+\hat{S}_{x}\right), \hat{H}(\boldsymbol{r})\right]} \\
& \quad=\left[\left(\hat{L}_{x}+\frac{\hbar}{2} \hat{\alpha}\right), \hat{H}(\boldsymbol{r})\right]=\mathrm{i} \hbar c\left(\hat{p}_{z} \hat{\beta}-\hat{p}_{y} \hat{\chi}\right) \\
& \quad+\frac{\hbar}{2}(-2 \mathrm{i} c)\left(\hat{p}_{z} \hat{\beta}-\hat{p}_{y} \hat{\chi}\right)=0 .
\end{aligned}
$$

For lower signs in formulae (3.2) - this corresponds to negative photon energies, i.e. corresponds to $\hat{H}(-\boldsymbol{r})$, we have $\left[\hat{J}_{x}, \hat{H}(-\boldsymbol{r})\right]=0$. It can be proved, in the same manner, that both $y$ and $z$ components of total momentum $\hat{\boldsymbol{J}}=\hat{\boldsymbol{L}}+\hat{\boldsymbol{S}}$ commute with photon Hamiltonian (the expression $(2.5)$ with sign + , i.e. $\hat{H}(\boldsymbol{r})$, will be called photon Hamiltonian). In the same time, the expression (2.5) with sign -, i.e. $\hat{H}(-\boldsymbol{r})$, will be called antiphoton Hamiltonian. The final conclusion is the following: total angular momentum $\hat{\boldsymbol{L}}+\hat{\boldsymbol{S}}$ is the integral of motion for photon. Up to now we have the proof that total angular momentum $\hat{\boldsymbol{L}}+\hat{\boldsymbol{S}}$ is free photon integral of motion, for arbitrary spin.

In nonrelativistic quantum mechanic the conclusion that $\hat{\boldsymbol{J}}$ is integral of motion would mean that energy and total momentum of the quantum object can be measured simultaneously and exactly. Since photon is relativistic object [5] the maximal exactness of measuring of photon momentum is given by $\Delta p \Delta t \sim \hbar / c$, and consequently 
energy and total momentum can be determined with an error of the order $\Delta E \Delta t \sim \hbar$. The orbital angular momentum $\hat{L}$, as it follows from (3.2), is not the integral of motion, but for relativistic object this fact is not essential, since for relativistic object absolutely exact determining of physical characteristics is impossible.

Considering the correspondence (3.3), photon Hamiltonian which is given by $\hat{H}=c\left(\hat{\alpha} \hat{p}_{x}+\hat{\beta} \hat{p}_{y}+\hat{\chi} \hat{p}_{z}\right)$ can be expressed by means of spin operators in the following form:

$$
\hat{H}=\frac{2 c}{\hbar}\left(\hat{S}_{x} \hat{p}_{x}+\hat{S}_{y} \hat{p}_{y}+\hat{S}_{z} \hat{p}_{z}\right) .
$$

The obtained form of photon Hamiltonian, which includes operators of translation moment $\hat{\boldsymbol{P}}$ and $\operatorname{spin} \hat{\boldsymbol{S}}$ suggests that a free photon has wealthy internal dynamics that consists of mutual action of its translation and spin characteristics. This "internal life" will be examined further in the paper.

\section{Unitary transformation of photon Hamiltonian}

Photon Hamiltonian (3.5) represents bilinear form in which photon momentum operators are multiplied by spin operators. Since momentum characterizes translation motion of photons, and spin characterizes rotation, it is obvious that the internal dynamic structure of a photon is determined by both its translation and rotation characteristics, and that their interaction - considering the form of Hamiltonian (3.10), leads to hybridization of excitations [6].

Spin operators in (3.5) correspond to spin $S=1 / 2$. Spin operators can then be represented by Pauli's operators in the following manner [7]:

$$
\begin{aligned}
& \hat{S}_{x}-\mathrm{i} \hat{S}_{y}=\hbar P^{+}, \quad \hat{S}_{x}+\mathrm{i} \hat{S}_{y}=\hbar P, \\
& \frac{1}{2}-\hat{S}_{z}=\hbar P^{+} P .
\end{aligned}
$$

On the other hand, Pauli's operators fulfill following commutation relations:

$$
\begin{aligned}
& {\left[P_{i}, P_{j}^{+}\right]=\left[1-2 P_{i}^{+} P_{j}\right] \delta_{i j},} \\
& {\left[P_{i}, P_{j}\right]=\left[P_{i}^{+}, P_{j}^{+}\right]=0,} \\
& P_{i}^{2}=P_{i}^{+2}=0,\left(P^{+} P\right)_{\mathrm{e} . \mathrm{v}}=\left\{\begin{array}{l}
0, \\
1 .
\end{array}\right.
\end{aligned}
$$

After substitution of (4.1) in (3.7) (where sign + is retained), we obtain the following Hamiltonian:

$$
\begin{aligned}
& \hat{H}=c \hat{p}_{z}+c\left[\left(\hat{p}_{x}-\mathrm{i} \hat{p}_{y}\right) P+\left(\hat{p}_{x}+\mathrm{i} \hat{p}_{y}\right) P^{+}\right. \\
& \left.\quad-2 \hat{p}_{z} P^{+} P\right] .
\end{aligned}
$$

This conversion to Pauli operators has been made because the physical picture of processes is clearer through creation and annihilation operators of excitation.

Operators of moments are linear in operators of creation and annihilation of photon: $P \sim A+A^{+}$, so it can easily be concluded that mean value of photon Hamiltonian over states $\frac{1}{n !}\left(A^{+}\right)^{n} P^{+}|0\rangle$ is equal to zero. This means that the method of theory of perturbation would be inappropriate for Hamiltonian (4.3) analysis. This is why we would make unitary transformation of photon Hamiltonian with the goal to bring it into the form more suitable for calculation than the form (4.3), i.e. we shall go to equivalent Hamiltonian given by

$$
\begin{aligned}
& \hat{H}_{\mathrm{eq}}=\mathrm{e}^{\hat{W}} \hat{H} \mathrm{e}^{-\hat{W}}, \\
& \hat{W}=\mathrm{i} \boldsymbol{k} \boldsymbol{r}+\rho\left(P-P^{+}\right)+\mathrm{i} \lambda P^{+} P,
\end{aligned}
$$

where $\rho$ and $\lambda$ are real parameters. Equivalent Hamiltonian is found using Weil's identity [8]:

$$
\begin{aligned}
& \mathrm{e}^{\hat{W}} \hat{D} \mathrm{e}^{-\hat{W}} \\
& \quad=\sum_{n=0}^{\infty} \frac{(1)^{n}}{n !} \underbrace{[\hat{W},[\hat{W}, \ldots[\hat{W}, \ldots[\hat{W}, \hat{D}]] \ldots]}_{n \text {-times }} .
\end{aligned}
$$

It includes the terms of the following type: $P+P^{+}$, $P-P^{+}$and $P^{+} P$. Undetermined parameter $\lambda$ has been determined so that the term proportional to $P-P^{+}$disappears from equivalent Hamiltonian. The final result of the described procedure is as follows:

$$
\begin{aligned}
& \hat{H}_{\mathrm{eq}}=E_{0}+\hat{H}+\hat{H}_{\mathrm{S}}, \quad E_{0}=\hbar c\left(k_{x} \sin 2 \rho+k_{z} \cos 2 \rho\right), \\
& \hat{H}_{\mathrm{S}}=-g\left(P+P^{+}\right)+2 a P^{+} P, \\
& g=\hbar c \sqrt{k_{y}^{2}+k_{x}^{2} \cos ^{2} 2 \rho+k_{z}^{2} \sin ^{2} 2 \rho-k_{x} k_{z} \sin 4 \rho,} \\
& a=\hbar c\left(k_{x} \sin 2 \rho+k_{z} \cos 2 \rho\right) .
\end{aligned}
$$

In the further we shall analyze free photon behavior using method of Green's functions [7-10]. It is important that Hamiltonian $E_{0}$ is irrelevant in Green's function techniques. Besides of this, the starting Hamiltonian $\hat{H}$, as we have already concluded earlier, has zero mean value over states $\frac{1}{n !}\left(A^{+}\right)^{n} P^{+}|0\rangle$. This is why we shall exclude it from calculations. The analysis of photon internal processes will be made with Hamiltonian $\hat{H}_{\mathrm{S}}$.

\section{Photon's ordering}

Since Pauli's operators figure in $\hat{H}_{\mathrm{S}}$ Hamiltonian without various configuration indices, the analysis of spin processes in a free photon will be made by means of anticommutator's Pauli's Green's function

$$
\begin{aligned}
& \Gamma(t)=\left\langle\left\langle P(t) \mid P^{+}(0)\right\rangle\right\rangle \\
& \quad=\Theta(t)\left\langle P(t) P^{+}(0)+P^{+}(0) P(t)\right\rangle,
\end{aligned}
$$

where $\Theta(t)$ is Heaviside's step-function [7-9]. Correlator of anticommutator's Pauli's Green's function contains mean value of anticommutator of Pauli's operator of the same configuration index, and according to (4.2) it is equal to one. This fact simplifies evaluation of mean values by means of spectral intensity of Green's function. 
Differentiating $\Gamma(t)$ with respect to time and using equation of motion for operator $P$, we come to

$$
\mathrm{i} \hbar \frac{\mathrm{d} \Gamma(t)}{\mathrm{d} t}=\mathrm{i} \hbar \delta(t)+2 a \Gamma(t)+2 g \Delta(t) .
$$

The Green functions of the type: $\left\langle\left\langle\right.\right.$ const $\left.\left.\mid P^{+}\right\rangle\right\rangle$are equal to zero. The function $\Delta(t)$ is given by

$$
\Delta(t)=\left\langle\left\langle P^{+}(t) P(t) \mid P^{+}(0)\right\rangle\right\rangle .
$$

Using the same procedure, for function $\Delta(t)$ we obtain the following equation:

$$
\begin{aligned}
& \mathrm{i} \hbar \frac{\mathrm{d} \Delta(t)}{\mathrm{d} t}=g \Gamma(t)-g F(t), \quad F(t)=\left\langle\left\langle P^{+}(t) \mid P^{+}(0)\right\rangle\right\rangle \\
& \rightarrow \mathrm{i} \hbar \frac{\mathrm{d} F(t)}{\mathrm{d} t}=-2 g \Delta(t)-2 a F(t) .
\end{aligned}
$$

In differential equations (5.2) and (5.4), Furrier transformations time-frequency are then made: $f(t)=\int_{-\infty}^{+\infty} \mathrm{d} t \mathrm{e}^{-\mathrm{i} \omega t} f(\omega), \quad f \equiv(\Gamma, \Delta, F), \delta(t)=$ $\frac{1}{2 \pi} \int_{-\infty}^{+\infty} \mathrm{d} t \mathrm{e}^{-\mathrm{i} \omega t}$, so we obtain the system of three algebraic equations

$$
\begin{aligned}
& (E-2 a) \Gamma(\omega)-2 g \Delta(\omega)=\frac{\mathrm{i} \hbar}{2 \pi}, \\
& \Delta(\omega)=g[\Gamma(\omega)-F(\omega)], \\
& E F(\omega)=-2[g \Delta(\omega)+a F(\omega)] .
\end{aligned}
$$

Solving this system of equations, we find that

$$
\begin{aligned}
& \Gamma(\omega)=\frac{i \hbar}{2 \pi} \frac{E^{2}+2 a E-2 g^{2}}{\left(E^{2}-E_{0}^{2}\right)^{2}}, \\
& E_{0}=2 \sqrt{a^{2}+g^{2}}=2 \hbar c k .
\end{aligned}
$$

In order to determine spectral intensity of function $\Gamma$, it is necessary to break down the right side of the formula (5.1) into common fractions. So, we obtain the following:

$$
\begin{aligned}
& \Gamma(\omega)=\frac{\mathrm{i}}{2 \pi}\left[\frac{2 g^{2}}{E_{0}^{2}} \frac{1}{\omega}+\left(\frac{1}{2}-\frac{g^{2}}{E_{0}^{2}}+\frac{a}{E_{0}}\right) \frac{1}{\omega-\omega_{0}}\right. \\
& \left.+\left(\frac{1}{2}-\frac{g^{2}}{E_{0}^{2}}-\frac{a}{E_{0}}\right) \frac{1}{\omega+\omega_{0}}\right],
\end{aligned}
$$

where $\omega=E / \hbar$ and $\omega_{0}=E_{0} / \hbar$. Since function $\Gamma$ is anticommutator's function, its spectral intensity is given by the formula [7]:

$$
I_{\Gamma}(\omega)=\frac{\Gamma(\omega+\mathrm{i} \delta)+\Gamma(\omega-\mathrm{i} \delta)}{\mathrm{e}^{\frac{\hbar \omega}{k_{\mathrm{B}} T}}+1}, \quad \delta \rightarrow+0,
$$

and using Dirac's formula: $\left(\omega-\omega_{k} \pm \mathrm{i} \delta\right)^{-1}=$ P.V. $\left\{\left(\omega-\omega_{k}\right)^{-1}\right\} \mp \mathrm{i} \pi \delta\left(\omega-\omega_{k}\right)$, where P.V. denotes principal value of integral, we find the explicit expression for spectral intensity:

$$
\begin{aligned}
& I_{\Gamma}(\omega)=\frac{2 g^{2}}{E_{0}^{2}} \frac{\delta(\omega)}{\mathrm{e}^{\hbar \omega / k_{\mathrm{B}} T}+1} \\
& \quad+\left(\frac{1}{2}-\frac{g^{2}}{E_{0}^{2}}+\frac{a}{E_{0}}\right) \frac{\delta\left(\omega-\omega_{0}\right)}{\mathrm{e}^{\hbar \omega / k_{\mathrm{B}} T}+1}
\end{aligned}
$$

$$
+\left(\frac{1}{2}-\frac{g^{2}}{E_{0}^{2}}-\frac{a}{E_{0}}\right) \frac{\delta\left(\omega+\omega_{0}\right)}{\mathrm{e}^{\hbar \omega / k_{\mathrm{B}} T}+1} .
$$

Now we can define the expression for correlation function of a free photon as

$$
\begin{aligned}
& \left\langle P^{+}(0) P(t)\right\rangle \equiv \int_{-\infty}^{+\infty} \mathrm{d} \omega \mathrm{e}^{-\mathrm{i} \omega t} I_{\Gamma}(\omega) \\
& =\frac{2 g^{2}}{E_{0}^{2}} \frac{1}{2}+\left(\frac{1}{2}-\frac{g^{2}}{E_{0}^{2}}+\frac{a}{E_{0}}\right) \frac{\mathrm{e}^{-\mathrm{i} \omega_{0} t}}{\mathrm{e}^{\hbar \omega_{0} / k_{\mathrm{B}} T}+1} \\
& +\left(\frac{1}{2}-\frac{g^{2}}{E_{0}^{2}}-\frac{a}{E_{0}}\right) \frac{\mathrm{e}^{\mathrm{i} \omega_{0} t}}{\mathrm{e}^{\hbar \omega_{0} / k_{\mathrm{B}} T}+1} .
\end{aligned}
$$

Next, we can calculate expression for concentration of spin excitations of a free photon. It is obtained from (5.11), if we take in it that $t=0$, i.e. $\left\langle P^{+} P\right\rangle=\frac{1}{2}-\frac{a}{E_{0}} \tanh \frac{\hbar c k}{k_{\mathrm{B}} T}$. Combining formulae for $a$ over formula (4.6), and $E_{0}$ from (5.6), and converting to sphere coordinate system, we find that $\frac{a}{E_{0}}=$ $\frac{1}{2}(\sin 2 \rho \sin \theta \cos \varphi+\cos 2 \rho \cos \theta)$. In accordance with this, we get the following expression for ordering parameter of spin subsystem in a free photon:

$$
\begin{aligned}
\sigma & =1-2\left\langle P^{+} P\right\rangle \\
& =(\sin 2 \rho \sin \theta \cos \varphi+\cos 2 \rho \cos \theta) \tanh \frac{\hbar c k}{k_{\mathrm{B}} T}
\end{aligned}
$$

The set of results of this section requires some explanations. The most interesting result is that energy for spin translation from $\hbar / 2$ to $-\hbar / 2$ is $2 \hbar c k$. This can be explained on the basis of measuring process in which incident photon bean is reflected by measuring devices. The angular momentum of incident phonon is $\hbar k$ while of reflected phonon is $-\hbar k$. So we obtain the change of photon momentum $\Delta p=\hbar k-(-\hbar k)=2 \hbar k$, and consequently the energy change $\Delta E=2 \hbar c k$. The energy $-\hbar c k$ corresponds to antiphoton, so that we can consider the described process as a transformation of photon to antiphoton. In this process the spin change takes place, also the Green function $\Gamma(t)=\left\langle\left\langle P(t) \mid P^{+}(0)\right\rangle\right\rangle$ was calculated. Since photon and antiphoton spins have opposite signs the change of the spin is $\Delta S=\hbar / 2-(-\hbar / 2)=\hbar$. The value of $\Delta S$ is equal to $\hbar$ and this is eigenvalue of spin $S=1$. This is the reason for behaving of photon as particle with spin $S=1$. The polar and azimuthal dependences of ordering parameter comes from the fact that incident beam must not be always orthogonal to the plane of measuring device.

\section{Conclusion}

Concluding the exposed analysis we shall try to connect the results obtained in series of experimental investigation of photon orbital angular momentum [11-20]. We did not describe all quoted experiments. Instead of it we described the essential idea: the orbital momentum of photon was determined from the changes of torque of rotating particles. These changes lied in some interval, so that the values of orbital angular momentum have had 
dispersion. As it was said at the end of first section, such result is expectable for relativistic objects, in this case for photons. The azimuthal dependence of measured results is also predicted by the theory exposed in Sect. 5 .

\section{Acknowledgments}

Investigations whose results are presented in this paper were partially supported by the Serbian Ministry of Sciences (grant No. 141044A) and by the Ministry of Sciences of Republic of Srpska.

\section{References}

[1] A. Messiah, Quantum Mechanics, North-Holland, Amsterdam 1970.

[2] P.A.M. Dirac, Principles of Quantum Mechanics, 4 ed., Univ. Press, Oxford 1958.

[3] M. Sapaznjikov, Anti-World - a Reality, Znanie, Moscow 1983 (in Russian), p. 33, 34.

[4] A.S. Davydov, Quantum Mechanics, Pergamon, London 1976.

[5] V.B. Berestetskii, E.M. Lipshitz, L.P. Pitaevskii, Quantum Electrodynamics, Pergamon, Oxford/New York 1982

[6] V.M. Agranovich, Theory of Excitons, Nauka, Moscow 1968 (in Russian).

[7] S.V. Tyablikov, Methods in the Quantum Theory in Magnetism, Plenum, New York (1967).
[8] B.S. Tošić, Statistical Physics, Faculty of Sciences, Novi Sad 1976 (in Serbian).

[9] G. Rickayzen, Green's Functions and Condensed Matter, Academic Press, London 1980.

[10] G. Mahan, Many Particle Physics, Plenum Press, New York 1990

[11] R.A. Beth, Phys. Rev. 50, 115 (1936).

[12] J. Leach, M.J. Padgett, S.M. Barnett, S. Franke-Amold, J. Courtial, Phys. Rev. Lett. 25, 257901 (2002).

[13] L. Allen, M.W. Beijersbergen, R.J.C. Spreeuw, J.P. Woerdman, Phys. Rev. A 45, 8185 (1992).

[14] P.J. Allen, Am. J. Phys. 74, 1185 (1966).

[15] H. He, M.E.J. Friese, N.R. Heckenberg, H. Rubinsztein-Dunlop, Phys. Rev. Lett. 75, 826 (1995).

[16] M.E.J. Friese, J. Enger, H. Rubinsztein-Dunlop, N.R. Heckenberg, Phys. Rev. A 54, 1593 (1996).

[17] S.J.van Enk, G. Nienhuis, Opt. Commun. 94, 147 (1992).

[18] E. Santamato, B. Daino, M. Romagnoli, M. Settembre, Y.R. Shen, Mol. Cryst. Liq. Cryst. 143, 89 (1987).

[19] A.T. O'Neil, I. Mac Vicar, L. Allen, M.J. Padgett, Phys. Rev. Lett. 88, 053601 (2002).

[20] K. Volke-Sepulveda, V. Garcés-Chávez, S. Chávez-Cerda, J. Arlt, K. Dholakia, J. Opt. B 4, S82 (2002). 